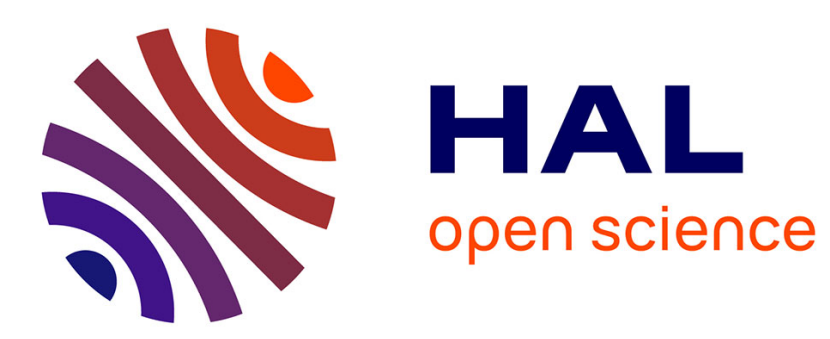

\title{
Error estimation of stress intensity factors for mixed-mode cracks
}

L. Gallimard, Julien Panetier

\section{To cite this version:}

L. Gallimard, Julien Panetier. Error estimation of stress intensity factors for mixed-mode cracks. International Journal for Numerical Methods in Engineering, 2006, 68 (3), pp.299-316. 10.1002/nme.1705 . hal-00109713

\section{HAL Id: hal-00109713 https://hal.science/hal-00109713}

Submitted on 7 Mar 2019

HAL is a multi-disciplinary open access archive for the deposit and dissemination of scientific research documents, whether they are published or not. The documents may come from teaching and research institutions in France or abroad, or from public or private research centers.
L'archive ouverte pluridisciplinaire HAL, est destinée au dépôt et à la diffusion de documents scientifiques de niveau recherche, publiés ou non, émanant des établissements d'enseignement et de recherche français ou étrangers, des laboratoires publics ou privés. 


\title{
Error estimation of stress intensity factors for mixed-mode cracks
}

\author{
L. Gallimard ${ }^{*} \dagger$ and J. Panetier \\ LMT-Cachan, ENS cachan/CNRS/Université Paris 6, 61 avenue du Président Wilson, \\ 94235 Cachan Cedex, France
}

\begin{abstract}
SUMMARY
This paper presents an a posteriori error estimator for mixed-mode stress intensity factors in plane linear elasticity. A surface integral over an arbitrary crown is used for the separate calculation of the combined mode's stress intensity factors. The error in the quantity of interest is based on goaloriented error measures and estimated through an error in the constitutive relation. Copyright (c) 2006 John Wiley \& Sons, Ltd.
\end{abstract}

KEY WORDS: finite element method; stress intensity factors; fracture; goal-oriented error estimation

\section{INTRODUCTION}

The development of a finite element discretized model starting from a continuous mechanical model leads to a partial loss of the information contained in the continuous model and, thus, to the introduction of discretization errors. Methods have been developed over many years to evaluate the global quality of finite element analyses [1-3]. For linear problems, all of these methods provide a global energy-based estimate of the discretization error. Most of the time, such global information is insufficient for dimensioning purposes in mechanical design. In many common situations, the dimensioning criteria involve local quantities (stresses, displacements, intensity factors...). The development of error estimators for such quantities was initiated in the 80's $[4,5]$. Recently, numerous works have been published which provide error estimates and bounds for several quantities of interest [6-12]. In these papers, the quantities of interest depend linearly on the solution of the problem and the calculation of the error estimate for the quantity of interest requires the approximate resolution of an auxiliary problem.

\footnotetext{
*Correspondence to: L. Gallimard, LMT-Cachan, ENS cachan/CNRS/Université Paris 6, 61 avenue du Président Wilson, 94235 Cachan Cedex, France.

†E-mail: laurent.gallimard@1mt.ens-cachan.fr

†Please ensure that you use the most up to date class file, available from the NME Home Page at http://www. interscience.wiley.com/jpages/0029-5981/
} 
Within the framework of the fracture mechanic, the stress intensity factors, the energy release rate $G$ and the $J$-integral are some of the well known quantity of interest. Few work are devoted to the study of the quality of these quantities. An estimation of the quality of the stress intensity factors was proposed in [13]. The approach was based on the partition of the error into two parts called local and pollution error and by constructing separate estimates for each component. However, this approach does not lead to lower or upper bounds of the stress intensity factors. The quality of the $J$-integral was studied in [14-16] by applying the techniques suggested in $[6,7]$. However, as the $J$-integral is a quadratic functional of the displacement, the proposed estimators are based on a linearisation of the $J$-integral. Recently in [17] it was proposed lower and higher bounds of this quantity by introducing rigorous bounds of the quadratic part.

In this work, we propose an error estimator for mixed-mode stress intensity factors in plane linear elasticity. Contrary to the $J$-integral, the stress intensity factors have the advantage of being linear functions of displacement, and it is possible to directly apply the techniques developed in [6-12] to estimate their quality. The simplest way to calculate the stress intensity factors as linear functions of the solution of the problem is through the Crack Opening Displacement Method [18]. This method is easy to use and it enables one to obtain the stress intensity factors for a mixed-mode fracture problem with a very simple post-processing. However, this formula itself involves an approximation of the exact stress intensity factors; its accuracy depends on the choice of the points on the side on the crack, and reliable results require a refined finite element mesh. In order to obtain an exact representation formula for the calculation of the stress intensity factors $K_{\mathrm{I}}$ and $K_{\mathrm{II}}$, we used the contour integral proposed by Stern et al. [19]. This contour integral is recast as a linear functional of the displacement involving only area integration.

The paper is organized as follows: in Section 2, we briefly recall the basics of constitutive relation error estimators in linear elasticity. Section 3 introduces the local version of the error estimator for quantities of interest which depends linearly on the solution of the problem. In Section 4, we define the output functional which must be estimated. Finally, numerical examples which illustrate the good behaviour of the error estimator are proposed in Section 5 .

\section{THE ERROR IN CONSTITUTIVE RELATION}

\subsection{The problem to be solved}

Let us consider a $2 \mathrm{D}$ elastic structure defined in a domain $\Omega$ bounded by $\partial \Omega$ (Figure 1 ). The external actions on the structure are represented by a prescribed displacement $\underline{u}_{d}$ over a subset $\partial_{1} \Omega$ of the boundary, a surface force density $\underline{F}_{d}$ defined over $\partial_{2} \Omega=\partial \Omega-\bar{\partial}_{1} \Omega$, and a body force density $\underline{f}_{d}$ defined in $\Omega$. The Hooke's operator of the material is denoted $\mathbf{K}$. Thus, the problem can be formulated as: Find a displacement field $\underline{u}$ and a stress field $\sigma$ defined in $\Omega$ which verify:

- the kinematic constraints:

$$
\underline{u} \in \mathscr{U} \quad \text { and }\left.\quad \underline{u}\right|_{\partial_{1} \Omega}=\underline{u}_{d}
$$




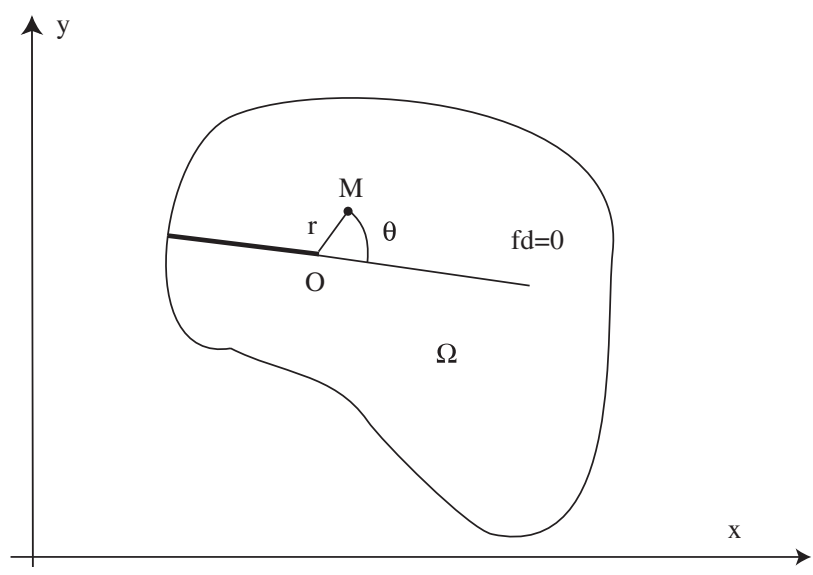

Figure 1. The structure $\Omega$.

- the equilibrium equations:

$$
\begin{gathered}
\boldsymbol{\sigma} \in \mathscr{S} \text { and } \forall \underline{u}^{*} \in \mathscr{U}_{0} \\
-\int_{\Omega} \boldsymbol{\sigma}: \boldsymbol{\varepsilon}\left(\underline{u}^{*}\right) \mathrm{d} \Omega+\int_{\Omega} \underline{f}_{d} \cdot \underline{u}^{*} \mathrm{~d} \Omega+\int_{\partial_{2} \Omega} \underline{F}_{d} \cdot \underline{u}^{*} \mathrm{~d} \Gamma=0
\end{gathered}
$$

- the constitutive relation:

$$
\boldsymbol{\sigma}=\mathbf{K} \varepsilon(\underline{u})
$$

$\mathscr{U}$ is the space in which the displacement field is being sought, $\mathscr{S}=\mathscr{L}_{2}[\Omega]^{3}$ the space of the stresses, $\mathscr{U}_{0}$ the space of the fields in $\mathscr{U}$ which are zero on $\partial_{1} \Omega$, and $\boldsymbol{\varepsilon}(\underline{u})$ denotes the linearized deformation associated with the displacement:

$$
[\boldsymbol{\varepsilon}(\underline{u})]_{i j}=\frac{1}{2}\left(u_{i, j}+u_{j, i}\right)
$$

The Galerkin finite element method provides an approximation $\underline{u}_{h}$ to $\underline{u}$ in a finite element space $\mathscr{U}_{h} \in \mathscr{U}$. The finite-dimension space $\mathscr{U}_{h}$ is associated with a finite element mesh of characteristic size $h$. Let $\mathscr{P}_{h}$ denote a partition of $\Omega$ into $N$ elements $E_{k}$. This partition is assumed to verify $\bar{\Omega}=\bigcup_{k} \bar{E}_{k}$ (the mesh cover the whole domain) with $E_{i} \cap E_{j}=\emptyset$ for any $i$ different from $j$. The discretized problem is:

Find a kinematically admissible finite element displacement field $\underline{u}_{h}$ such that:

$$
\begin{gathered}
\forall \underline{u}_{h}^{*} \in \mathscr{U}_{h 0} \\
-\int_{\Omega} \mathbf{K} \boldsymbol{\varepsilon}\left(\underline{u}_{h}\right): \boldsymbol{\varepsilon}\left(\underline{u}_{h}^{*}\right) \mathrm{d} \Omega+\int_{\Omega} \underline{f}_{d} \cdot \underline{u}_{h}^{*} \mathrm{~d} \Omega+\int_{\partial_{2} \Omega} \underline{F}_{d} \cdot \underline{u}_{h}^{*} \mathrm{~d} \Gamma=0
\end{gathered}
$$

where $\mathscr{U}_{h 0}=\left\{\underline{u}_{h} \in \mathscr{U}_{h} ;\left.\underline{u}_{h}\right|_{\partial_{1} \Omega}=\underline{0}\right\}$

The corresponding stress field is calculated using the constitutive relation:

$$
\boldsymbol{\sigma}_{h}=\mathbf{K \varepsilon}\left(\underline{u}_{h}\right)
$$


The discretization error $\underline{e}_{h}$ is the difference between the finite element displacement and the actual solution of the problem.

$$
\underline{e}_{h}=\underline{u}-\underline{u}_{h}
$$

Traditionally, the energy norm is used as the measure of the error:

$$
e_{h}=\left[\int_{\Omega} \mathbf{K} \boldsymbol{\varepsilon}\left(\underline{u}-\underline{u}_{h}\right): \boldsymbol{\varepsilon}\left(\underline{u}-\underline{u}_{h}\right) \mathrm{d} \Omega\right]^{1 / 2}=\left[\int_{\Omega}\left(\boldsymbol{\sigma}-\boldsymbol{\sigma}_{h}\right): \mathbf{K}^{-1}\left(\boldsymbol{\sigma}-\boldsymbol{\sigma}_{h}\right) \mathrm{d} \Omega\right]^{1 / 2}
$$

The contribution of an element $E$ of the finite element mesh to the global error is

$$
\begin{aligned}
e_{h E} & =\left[\int_{E} \mathbf{K} \boldsymbol{\varepsilon}\left(\underline{u}-\underline{u}_{h}\right): \boldsymbol{\varepsilon}\left(\underline{u}-\underline{u}_{h}\right) \mathrm{d} E\right]^{1 / 2} \\
& =\left[\int_{E}\left(\boldsymbol{\sigma}-\boldsymbol{\sigma}_{h}\right): \mathbf{K}^{-1}\left(\boldsymbol{\sigma}-\boldsymbol{\sigma}_{h}\right) \mathrm{d} E\right]^{1 / 2}
\end{aligned}
$$

with the relation:

$$
e_{h}^{2}=\sum_{E \in \mathscr{P}_{h}} e_{h E}^{2}
$$

\subsection{Definition of the error in constitutive relation}

The approach based on the error in constitutive relation relies on a partition of the equations of the problem to be solved into two groups. In linear elasticity, the first group consists of the kinematic constraints (1) and the equilibrium equations (2); the constitutive relation (3) constitutes the second group. Let us consider an approximate solution to the problem, denoted $(\underline{\hat{u}}, \hat{\boldsymbol{\sigma}})$, which verifies the first group of equations:

The fields $(\underline{\hat{u}}, \hat{\boldsymbol{\sigma}})$ are said to be admissible if:

- the field $\underline{\hat{u}}$ verifies (1),

- the field $\hat{\boldsymbol{\sigma}}$ verifies (2).

If $(\underline{\hat{u}}, \hat{\boldsymbol{\sigma}})$ verifies the constitutive relation (3) in $\Omega$, then $(\hat{\underline{u}}, \hat{\boldsymbol{\sigma}})=(\underline{u}, \boldsymbol{\sigma})$. If, however, $(\underline{\hat{u}}, \hat{\boldsymbol{\sigma}})$ does not verify the constitutive relation, the quality of this admissible solution is measured by the residual, denoted $\hat{\mathbf{e}}(\underline{\hat{u}}, \hat{\boldsymbol{\sigma}})$, with respect to the verification of the constitutive relation:

$$
\hat{\mathbf{e}}(\underline{\hat{u}}, \hat{\boldsymbol{\sigma}})=\hat{\boldsymbol{\sigma}}-\mathbf{K \varepsilon}(\underline{\hat{u}})
$$

The associated constitutive relation error for all the elements $E$ of the finite element mesh is

$$
\hat{e}^{2}(\underline{\hat{u}}, \hat{\boldsymbol{\sigma}})^{2}=\sum_{E \in \mathscr{P}_{h}} \hat{e}_{E}(\underline{\hat{u}}, \hat{\boldsymbol{\sigma}})^{2}
$$

where $\hat{e}_{E}$ is the contribution of element $E$ of the mesh to the error:

$$
\hat{e}_{E}(\underline{\hat{u}}, \hat{\boldsymbol{\sigma}})^{2}=\int_{E}(\hat{\boldsymbol{\sigma}}-\mathbf{K} \varepsilon(\underline{\hat{u}})): \mathbf{K}^{-1}(\hat{\boldsymbol{\sigma}}-\mathbf{K} \boldsymbol{\varepsilon}(\underline{\hat{u}})) \mathrm{d} E
$$


Then, the relative error $\hat{\varepsilon}$ is defined by

$$
\hat{\varepsilon}(\underline{\hat{u}}, \hat{\boldsymbol{\sigma}})^{2}=\frac{\hat{e}(\underline{\hat{u}}, \hat{\boldsymbol{\sigma}})^{2}}{\int_{\Omega} \hat{\boldsymbol{\sigma}}^{*} \mathbf{K}^{-1} \hat{\boldsymbol{\sigma}}^{*} \mathrm{~d} \Omega}
$$

with

$$
\hat{\boldsymbol{\sigma}}^{*}=\frac{1}{2}(\hat{\boldsymbol{\sigma}}+\mathbf{K \varepsilon}(\underline{\hat{u}}))
$$

The pair $\left(\underline{u}_{h}, \boldsymbol{\sigma}_{h}\right)$ is not an admissible solution. In order to develop an error estimator based on the concept of error in the constitutive relation, we construct an admissible pair $\left(\underline{\hat{u}}_{h}, \hat{\boldsymbol{\sigma}}_{h}\right)$ from the finite element solution and from the data.

- Since the finite element displacement field verifies the kinematic constraints, one takes:

$$
\underline{\hat{u}}_{h}=\underline{u}_{h} \quad \text { in } \Omega
$$

- However, the stress field $\boldsymbol{\sigma}_{h}$ does not verify the equilibrium equations (2). Techniques to reconstruct equilibrated stress fields from $\boldsymbol{\sigma}_{h}$ and the data have been under development for several years and are described in References [20-22].

\subsection{Relation to the discretization error}

The error in the constitutive relation can be related to the discretization error through the Prager-Synge theorem [23]:

$$
\hat{e}_{h}^{2}=\int_{\Omega}\left(\hat{\boldsymbol{\sigma}}_{h}-\boldsymbol{\sigma}\right): \mathbf{K}^{-1}\left(\hat{\boldsymbol{\sigma}}_{h}-\boldsymbol{\sigma}\right) \mathrm{d} \Omega+\int_{\Omega}\left(\boldsymbol{\sigma}-\boldsymbol{\sigma}_{h}\right): \mathbf{K}^{-1}\left(\boldsymbol{\sigma}-\boldsymbol{\sigma}_{h}\right) \mathrm{d} \Omega
$$

This theorem leads to the following inequalities:

$$
e_{h}=\left[\int_{\Omega}\left(\boldsymbol{\sigma}-\boldsymbol{\sigma}_{h}\right): \mathbf{K}^{-1}\left(\boldsymbol{\sigma}-\boldsymbol{\sigma}_{h}\right) \mathrm{d} \Omega\right]^{1 / 2} \leqslant \hat{e}_{h}
$$

and

$$
\left[\int_{\Omega}\left(\hat{\boldsymbol{\sigma}}_{h}-\boldsymbol{\sigma}\right): \mathbf{K}^{-1}\left(\hat{\boldsymbol{\sigma}}_{h}-\boldsymbol{\sigma}\right) \mathrm{d} \Omega\right]^{1 / 2} \leqslant \hat{e}_{h}
$$

\section{GOAL-ORIENTED ERROR ESTIMATION}

Instead of estimating the numerical error in terms of the energy norm, it would be preferable to express this error in terms of physically meaningful quantities of interest. In this section, we briefly recall the techniques developed in the literature for the case where the quantity of interest is a linear functional $L$ of the displacement. In this case, the objective of the calculation is to assess the quality of $I_{h}=L\left(\underline{u}_{h}\right)$ by estimating $\left|I-I_{h}\right|$, where $I=L(\underline{u})$. We refer the reader to References [9-13] for a detailed description of this approach. Because of the linearity 
assumption, one obtains:

$$
I-I_{h}=L(\underline{u})-L\left(\underline{u}_{h}\right)=L\left(\underline{u}-\underline{u}_{h}\right)=L\left(\underline{e}_{h}\right)
$$

Thus, the estimation of $\left|I-I_{h}\right|$ is equivalent to the estimation of $\left|L\left(\underline{e}_{h}\right)\right|$.

\subsection{Definition of the auxiliary problem}

Let us consider the following auxiliary problem: Find $\underline{z} \in \mathscr{U}_{0}$ and $\boldsymbol{\sigma}^{\text {aux }}=\mathbf{K} \boldsymbol{\varepsilon}(\underline{z})$ such that:

$$
\forall \underline{u}^{*} \in \mathscr{U}_{0} \int_{\Omega} \mathbf{K} \boldsymbol{\varepsilon}\left(\underline{u}^{*}\right): \boldsymbol{\varepsilon}(\underline{z}) \mathrm{d} \Omega=L\left(\underline{u}^{*}\right)
$$

Replacing $\underline{u}^{*}$ by $\underline{e}_{h}$, one obtains

$$
L\left(\underline{e}_{h}\right)=\int_{\Omega} \mathbf{K} \boldsymbol{\varepsilon}\left(\underline{e}_{h}\right): \boldsymbol{\varepsilon}(\underline{z}) \mathrm{d} \Omega
$$

The function $\underline{z}$ indicates how the discretization error affects the quantity $L\left(\underline{e}_{h}\right)$. If $\underline{z}$ could be exactly computed, one could determine $L(\underline{u})$ directly from the input data, as from (2) and (18) one would have:

$$
L(\underline{u})=\int_{\Omega} \mathbf{K} \varepsilon(\underline{u}): \varepsilon(\underline{z}) \mathrm{d} \Omega=\int_{\Omega} \underline{f}_{d} \cdot \underline{z} \mathrm{~d} \Omega+\int_{\partial_{2} \Omega} \underline{F}_{d} \cdot \underline{z} \mathrm{~d} \Gamma
$$

Unfortunately, the problem for the function $z$ is as complicated to solve as the reference problem for the solution $\underline{u}$. Thus we have to compute an approximate value for $z$.

Relation (19) is the starting point of the goal-oriented error estimators developed in References $[5,6,8,9,11,13]$. The approaches proposed in these papers differ on three points:

- the technique developed to approximate $\underline{z}$,

- the technique used to obtain lower and upper bounds of $\left|L\left(\underline{e}_{h}\right)\right|$,

- the error estimator used to calculate the bounds.

\subsection{Approximate solution of the auxiliary problem}

Let $\underline{z}_{h}$ be the finite element approximation of $\underline{z}$ defined in the finite element space $\mathscr{U}_{h 0}$ :

$$
\forall \underline{u}_{h}^{*} \in \mathscr{U}_{h 0} \int_{\Omega} \mathbf{K} \boldsymbol{\varepsilon}\left(\underline{u}_{h}^{*}\right): \boldsymbol{\varepsilon}\left(\underline{z}_{h}\right) \mathrm{d} \Omega=L\left(\underline{u}_{h}^{*}\right)
$$

Because of the orthogonality property, one has:

$$
\int_{\Omega} \mathbf{K} \boldsymbol{\varepsilon}\left(\underline{e}_{h}\right): \boldsymbol{\varepsilon}\left(\underline{u}_{h}^{*}\right) \mathrm{d} \Omega=0 \quad \forall \underline{u}_{h}^{*} \in \mathscr{U}_{h 0}
$$

Replacing $\underline{u}_{h}^{*}$ by $\underline{z}_{h}$ :

$$
\int_{\Omega} \mathbf{K} \boldsymbol{\varepsilon}\left(\underline{e}_{h}\right): \boldsymbol{\varepsilon}\left(\underline{z}_{h}\right) \mathrm{d} \Omega=0
$$


By combining (19) and (22), one obtains

$$
I-I_{h}=L\left(\underline{e}_{h}\right)=\int_{\Omega} \mathbf{K} \boldsymbol{\varepsilon}\left(\underline{e}_{h}\right): \boldsymbol{\varepsilon}\left(\underline{z}_{-}-\underline{z}_{h}\right) \mathrm{d} \Omega=\int_{\Omega}\left(\boldsymbol{\sigma}^{\mathrm{aux}}-\boldsymbol{\sigma}_{h}^{\mathrm{aux}}\right): \boldsymbol{\varepsilon}\left(\underline{e}_{h}\right) \mathrm{d} \Omega
$$

Let $\hat{\boldsymbol{\sigma}}_{h}^{\text {aux }}$ be an equilibrated stress associated with $\boldsymbol{\sigma}_{h}^{\text {aux }}$ through the techniques developed in Reference [20]. The following property is verified:

$$
\int_{\Omega}\left(\boldsymbol{\sigma}^{\mathrm{aux}}-\hat{\boldsymbol{\sigma}}_{h}^{\mathrm{aux}}\right): \boldsymbol{\varepsilon}\left(\underline{u}^{*}\right) \mathrm{d} \Omega=0 \quad \forall \underline{u}^{*} \in \mathscr{U}_{0}
$$

In particular, replacing $\underline{u}^{*}$ by $\underline{e}_{h}$ :

$$
\int_{\Omega}\left(\boldsymbol{\sigma}^{\mathrm{aux}}-\hat{\boldsymbol{\sigma}}_{h}^{\mathrm{aux}}\right): \boldsymbol{\varepsilon}\left(\underline{e}_{h}\right) \mathrm{d} \Omega=0
$$

and combining (25) and (23):

$$
\begin{aligned}
I-I_{h}=L\left(\underline{e}_{h}\right) & =\int_{\Omega}\left(\hat{\boldsymbol{\sigma}}_{h}^{\mathrm{aux}}-\boldsymbol{\sigma}_{h}^{\mathrm{aux}}\right): \boldsymbol{\varepsilon}\left(\underline{e}_{h}\right) \mathrm{d} \Omega \\
& =\int_{\Omega}\left(\hat{\boldsymbol{\sigma}}_{h}^{\mathrm{aux}}-\boldsymbol{\sigma}_{h}^{\mathrm{aux}}\right): \mathbf{K}^{-1}\left(\boldsymbol{\sigma}-\boldsymbol{\sigma}_{h}\right) \mathrm{d} \Omega \\
& =\sum_{E \in \mathscr{P}_{h}} \int_{E}\left(\hat{\boldsymbol{\sigma}}_{h}^{\mathrm{aux}}-\boldsymbol{\sigma}_{h}^{\mathrm{aux}}\right): \mathbf{K}^{-1}\left(\boldsymbol{\sigma}-\boldsymbol{\sigma}_{h}\right) \mathrm{d} E
\end{aligned}
$$

\subsection{Upper-bound property}

Let $\hat{e}_{h}^{\text {aux }}$ be the constitutive relation error measured on the auxiliary problem:

$$
\left(\hat{e}_{h}^{\operatorname{aux}}\right)^{2}=\sum_{E \in \mathscr{P}_{h}}\left(\hat{e}_{h E}^{\operatorname{aux}}\right)^{2}
$$

with

$$
\left(\hat{e}_{h E}^{\mathrm{aux}}\right)^{2}=\hat{e}_{E}\left(\underline{z}_{h}, \hat{\boldsymbol{\sigma}}_{h}^{\mathrm{aux}}\right)^{2}=\int_{E}\left(\hat{\boldsymbol{\sigma}}_{h}^{\mathrm{aux}}-\boldsymbol{\sigma}_{h}^{\mathrm{aux}}\right): \mathbf{K}^{-1}\left(\hat{\boldsymbol{\sigma}}_{h}^{\mathrm{aux}}-\boldsymbol{\sigma}_{h}^{\mathrm{aux}}\right) \mathrm{d} E
$$

Applying the Cauchy-Schwartz inequality to relation (26), one obtains the following upper bounds:

$$
\left|I-I_{h}\right|=\hat{e}_{h}^{\text {aux }} e_{h} \cos \phi \leqslant \hat{e}_{h}^{\text {aux }} e_{h}
$$

where $\phi$ is the unknown angle between $\underline{z}-\underline{z}_{h}$ and $\underline{u}-\underline{u}_{h}$, and

$$
\left|I-I_{h}\right| \leqslant \sum_{E \in \mathscr{P}_{h}} \hat{e}_{h E}^{\text {aux }} e_{h E}
$$

Other bounds were developed in Reference [9]. 


\subsection{Goal-oriented error estimator}

By using the Prager-Synge theorem (16), relation (29) leads to

$$
\left|I-I_{h}\right| \leqslant \hat{e}_{h}^{\operatorname{aux}} \hat{e}_{h}
$$

However, this upper bound is too large to be of practical use when the angle $\phi$ becomes near $\pi / 2$. To overcome this problem, following the approach defined in References [10,22, 24], we estimate this bound using the error in constitutive relation developed in Reference [10]. The local error estimator is defined by

$$
\hat{e}_{\mathrm{loc}}=\sum_{E \in \mathscr{P}_{h}} \hat{e}_{h E}^{\mathrm{aux}} \hat{e}_{h E}
$$

where $\hat{e}_{h E}=\hat{e}_{E}\left(\underline{u}_{h}, \hat{\boldsymbol{\sigma}}_{h}\right)$ (Equation (12)), and $\hat{e}_{h E}^{\text {aux }}$ is defined by Equation (28).

The lower and upper bounds of the local quantity are estimated as

$$
\hat{I}_{h, \text { up }}=I_{h}+\hat{e}_{\mathrm{loc}} \quad \text { and } \quad \hat{I}_{h, \mathrm{lo}}=I_{h}-\hat{e}_{\mathrm{loc}}
$$

\section{APPLICATION TO THE ESTIMATION OF THE ERROR IN THE STRESS INTENSITY FACTORS}

Let us consider a two-dimensional cracked structure with no body forces (Figure 1). The faces $\Gamma_{1}$ and $\Gamma_{2}$ of the crack are traction-free and on the remainder of the boundary either the displacements or the tractions are prescribed.

The analytical expressions of the displacement near the crack's tip are known in polar coordinates:

$$
\begin{aligned}
u_{r}= & \frac{1}{4 \mu}\left(\frac{r}{2 \pi}\right)^{1 / 2}\left\{\left[(2 \kappa-1) \cos \frac{\theta}{2}-\cos \frac{3 \theta}{2}\right] K_{\mathrm{I}}\right. \\
& \left.-\left[(2 \kappa-1) \sin \frac{\theta}{2}-3 \sin \frac{3 \theta}{2}\right] K_{\mathrm{II}}\right\}+r^{1 / 2} \varepsilon(r) \\
u_{\theta}= & \frac{1}{4 \mu}\left(\frac{r}{2 \pi}\right)^{1 / 2}\left\{\left[-(2 \kappa+1) \sin \frac{\theta}{2}+\sin \frac{3 \theta}{2}\right] K_{\mathrm{I}}\right. \\
& \left.-\left[(2 \kappa+1) \cos \frac{\theta}{2}-3 \cos \frac{3 \theta}{2}\right] K_{\mathrm{II}}\right\}+r^{1 / 2} \varepsilon(r)
\end{aligned}
$$

with $\lim _{r \rightarrow 0} \varepsilon(r)=0$.

In this expression, $K_{\mathrm{I}}$ and $K_{\mathrm{II}}$ and the open-mode and shear-mode stress intensity factors, respectively; $\mu$ is the shear modulus and $\kappa$ a parameter which is equal to $(3-v) /(1+v)$ for plane stress and to $3-4 v$ for plane strain; $v$ denotes the Poisson's ratio. 


\subsection{Expression of the output quantities of interest $K_{I}$ and $K_{I I}$}

Maxwell-Betti's reciprocal work theorem for the plane elastic state leads to a path-independent contour integral, which was introduced in Reference [19]:

$$
\int_{\partial \omega}[\underline{u} . \tilde{\sigma} \underline{n}-\underline{\tilde{u}} \cdot \boldsymbol{\sigma} \underline{n}] \mathrm{d} s=0
$$

where $\partial \omega=C_{1} \cup \Gamma_{2}^{\varepsilon} \cup C_{\varepsilon} \cup \Gamma_{1}^{\varepsilon}$ and $(\underline{\tilde{u}}, \tilde{\boldsymbol{\sigma}})$ is an arbitrary solution of the elastic problem defined near the crack's tip:

$$
\begin{aligned}
\operatorname{div} \tilde{\boldsymbol{\sigma}} & =\underline{0} \quad \text { in } \omega_{1} \\
\tilde{\tilde{\boldsymbol{\sigma}}} \underline{n} & =0 \quad \text { on } \Gamma_{1}^{\varepsilon} \cup \Gamma_{2}^{\varepsilon} \\
\tilde{\boldsymbol{\sigma}} & =\mathbf{K} \boldsymbol{\varepsilon}(\underline{u}) \quad \text { in } \omega_{1}
\end{aligned}
$$

where $C_{\varepsilon}$ designates a circular boundary of radius $r_{\varepsilon}$ centred at the crack's tip and $C_{1}$ the remainder of the boundary, as shown in Figure 2.

Equation (35) can be written in the form:

$$
-\int_{C_{\varepsilon}}[\underline{u} . \tilde{\tilde{\sigma}} \underline{n}-\underline{\tilde{u}} \cdot \boldsymbol{\sigma} \underline{n}] \mathrm{d} s=\int_{C_{1}}[\underline{u} . \tilde{\tilde{\sigma}} \underline{n}-\underline{\tilde{u}} \cdot \boldsymbol{\sigma} \underline{n}] \mathrm{d} s
$$

Let us designates by $I_{\varepsilon}$ the contour integral on $C_{\varepsilon}$.

$$
I_{\varepsilon}(\underline{\tilde{u}}, \tilde{\boldsymbol{\sigma}})=-\int_{C_{\varepsilon}}[\underline{u} . \tilde{\tilde{\sigma}} \underline{n}-\underline{\tilde{u}} . \boldsymbol{\sigma} \underline{n}] \mathrm{d} s
$$

Equation (37) shows that the contour integral $I_{\varepsilon}(\tilde{u}, \tilde{\boldsymbol{\sigma}})$ is independent on the radius $r_{\varepsilon}$. The idea now is to evaluate these integrals for arbitraly small $r_{\varepsilon}$ and a suitable choice for the pair $(\underline{\tilde{u}}, \tilde{\boldsymbol{\sigma}})$. Stern et al. [19] proposed two solutions of Equation (36) defined by (39) and (40) such

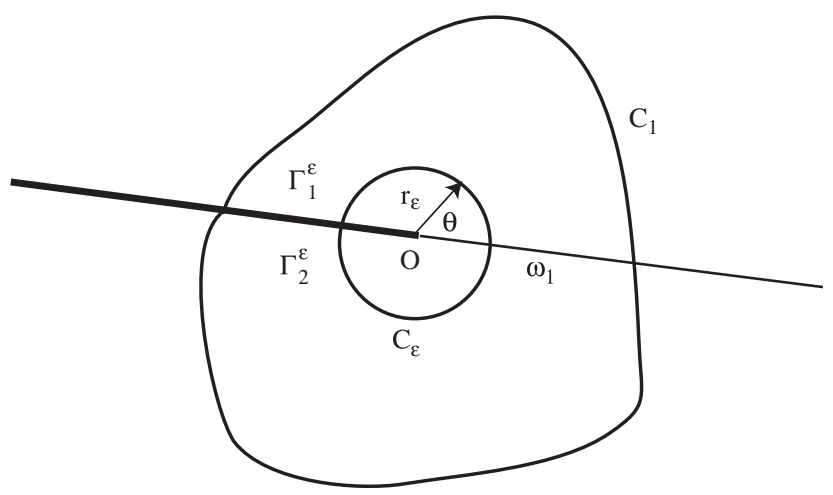

Figure 2. Around the crack's tip. 
that the products $r^{1 / 2} \underline{\tilde{u}}^{\bullet}$ and $r^{3 / 2} \tilde{\boldsymbol{\sigma}}^{\bullet}$ are finite on $C_{\varepsilon}($ where $\bullet=\mathrm{I}, \mathrm{II})$.

$$
\left\{\begin{array}{l}
\tilde{u}_{r}^{\mathrm{I}}=u_{0}\left((2 \kappa+1) \cos \frac{3 \theta}{2}-3 \cos \frac{\theta}{2}\right) \\
\tilde{u}_{\theta}^{\mathrm{I}}=u_{0}\left(-(2 \kappa-1) \sin \frac{3 \theta}{2}+3 \sin \frac{\theta}{2}\right) \\
\tilde{\sigma}_{r}^{\mathrm{I}}=\sigma_{0}\left(7 \cos \frac{3 \theta}{2}-3 \cos \frac{\theta}{2}\right) \\
\tilde{\sigma}_{\theta}^{\mathrm{I}}=\sigma_{0}\left(\cos \frac{3 \theta}{2}+3 \cos \frac{\theta}{2}\right) \\
\tilde{\sigma}_{r \theta}^{\mathrm{I}}=\sigma_{0}\left(3 \cos \frac{3 \theta}{2}+3 \cos \frac{\theta}{2}\right) \\
\tilde{\sigma}_{r \theta}^{\mathrm{II}}=-\sigma_{0}\left(\cos \frac{3 \theta}{2}+\cos \frac{\theta}{2}\right) \\
\tilde{u}_{r}^{\mathrm{II}}=u_{0}\left((2 \kappa+1) \sin \frac{3 \theta}{2}-\sin \frac{\theta}{2}\right) \\
\tilde{\sigma}_{r}^{\mathrm{II}}=\sigma_{\theta}\left(7 \sin \frac{3 \theta}{2}-\sin \frac{\theta}{2}\right) \\
\tilde{u}_{\theta}^{\mathrm{II}}=u_{0}\left((2 \kappa-1) \cos \frac{3 \theta}{2}-\cos \frac{\theta}{2}\right)
\end{array}\right.
$$

where $u_{0}=1 / 2(2 \pi r)^{1 / 2}(1+\kappa)$ and $\sigma_{0}=-\mu / 2\left(2 \pi r^{3}\right)^{1 / 2}(1+\kappa)$.

The evaluation of the $I_{\varepsilon}\left(\underline{\tilde{u}}^{\bullet}, \tilde{\boldsymbol{\sigma}}^{\bullet}\right)$ integral is performed on the inner circular boundary $C_{\varepsilon}$ :

$$
I_{\varepsilon}\left(\tilde{\tilde{u}}^{\bullet}, \tilde{\boldsymbol{\sigma}}^{\bullet}\right)=\int_{-\pi}^{+\pi}\left(\tilde{\sigma}_{r}^{\bullet} u_{r}+\tilde{\sigma}_{r \theta}^{\bullet} u_{\theta}-\sigma_{r} \tilde{u}_{r}^{\bullet}-\sigma_{r \theta} \tilde{u}_{\theta}^{\bullet}\right) r_{\varepsilon} \mathrm{d} \theta
$$

Introducing Equations (39) and (40) and the classical expressions of $u_{r}, u_{\theta}, \sigma_{r}, \sigma_{\theta}, \sigma_{r \theta}$ (Equation (34)) into Equation (41), one obtains:

$$
I_{\varepsilon}\left(\underline{\tilde{u}}^{\mathrm{I}}, \tilde{\boldsymbol{\sigma}}^{\mathrm{I}}\right)=K_{\mathrm{I}}+\varepsilon\left(r_{\varepsilon}\right) \quad \text { and } \quad I_{\varepsilon}\left(\underline{\tilde{u}}^{\mathrm{II}}, \tilde{\boldsymbol{\sigma}}^{\mathrm{II}}\right)=K_{\mathrm{II}}+\varepsilon\left(r_{\varepsilon}\right)
$$


where the last term $\varepsilon\left(r_{\varepsilon}\right)$ goes to zero. Relation (37) leads to the formula:

$$
\begin{aligned}
K_{\mathrm{I}} & =\int_{C_{1}}\left[\underline{u} \cdot \tilde{\boldsymbol{\sigma}}^{\mathrm{I}} \underline{n}-\underline{\tilde{u}}^{\mathrm{I}} \cdot \boldsymbol{\sigma} \underline{n}\right] \mathrm{d} s \\
K_{\mathrm{II}} & =\int_{C_{1}}\left[\underline{u} \cdot \tilde{\sigma}^{\mathrm{II}} \underline{n}-\underline{\tilde{u}}^{\mathrm{II}} \cdot \boldsymbol{\sigma} \underline{n}\right] \mathrm{d} s
\end{aligned}
$$

This path-independent integral cannot be used directly as the loading of an auxiliary problem because of the presence of the term $\int_{C} \underline{\tilde{u}} \cdot \sigma \underline{n} \mathrm{~d} s=\int_{C} \underline{\tilde{u}} .(\mathbf{K \varepsilon}(\underline{u})) \underline{n} \mathrm{~d} s$ which cannot written as a loading term. However, it can be transformed into a surface integral over an arbitrary crown $\omega_{2}$ by introducing an arbitrary scalar field $\phi$, continuously derivable over the crown $\omega_{2}$, such that $\phi(M)=1$ on $C_{1}$ and $\phi(M)=0$ on $C_{2}$. For the sake of simplicity, let us assume that $C_{1}$ and $C_{2}$ are circles centred at the crack's tip with radii $R_{1}$ and $R_{2}$, as shown in Figure 3. The function $\phi$ being used is

$$
\begin{aligned}
\phi(r, \theta) & =\frac{R_{2}-r}{R_{2}-R_{1}} \\
K_{\bullet} & =\int_{C_{1}}\left[\underline{u} \cdot \tilde{\tilde{\sigma}}^{\bullet} \underline{n}-\underline{\tilde{u}}^{\bullet} \cdot \boldsymbol{\sigma} \underline{n}\right] \phi \mathrm{d} s
\end{aligned}
$$

where $\bullet=$ I, II. Applying the divergence theorem, Equation (45) becomes:

$$
K_{\bullet}=\int_{\omega_{2}}\left(\mathbf{K} \boldsymbol{\varepsilon}\left(\phi \underline{\tilde{u}}^{\bullet}\right)-\phi \tilde{\boldsymbol{\sigma}}^{\bullet}\right): \boldsymbol{\varepsilon}(\underline{u}) \mathrm{d} \omega-\int_{\omega_{2}} \tilde{\boldsymbol{\sigma}}^{\bullet} \underline{\operatorname{grad}}(\phi) \cdot \underline{u} \mathrm{~d} \omega
$$

Thus, the stress intensity factors are expressed in the form of a linear output functional of the displacements $\underline{u}$. The approximate values $K_{\mathrm{I}, h}$ and $K_{\mathrm{II}, h}$ are obtained from the finite element solution by replacing the exact displacement field $\underline{u}$ by the finite element displacement

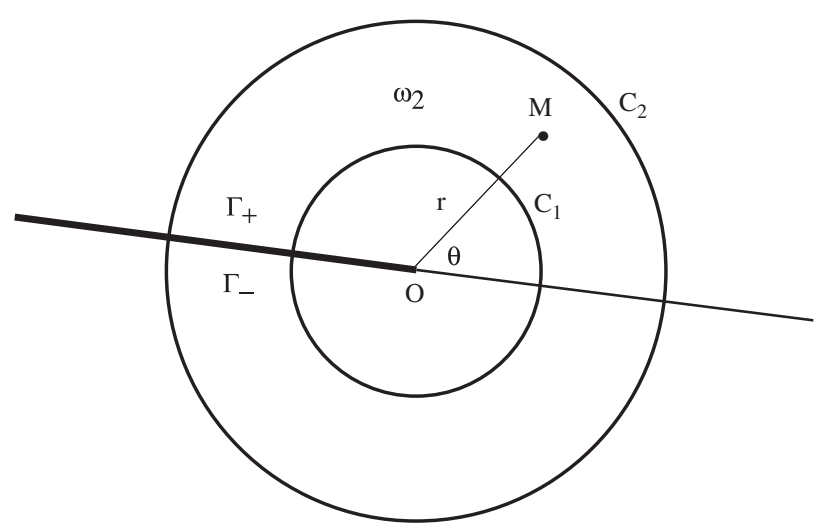

Figure 3. Arbitrary crown $\omega_{2}$. 
field $\underline{u}_{h}$ in relation (46):

$$
K_{\bullet}, h=\int_{\omega_{2}}\left(\mathbf{K} \boldsymbol{\varepsilon}\left(\phi \underline{\tilde{u}}^{\bullet}\right)-\phi \tilde{\boldsymbol{\sigma}}^{\bullet}\right): \boldsymbol{\varepsilon}\left(\underline{u}_{h}\right) \mathrm{d} \omega-\int_{\omega_{2}} \tilde{\tilde{\boldsymbol{\sigma}}}^{\bullet} \underline{\operatorname{grad}}(\phi) \cdot \underline{u}_{h} \mathrm{~d} \omega
$$

The error on these two linear functionals can be computed by using the techniques described in Section 3. The loading of the auxiliary problems are

$$
\begin{aligned}
& \text { a prestress }\left(\mathbf{K} \boldsymbol{\varepsilon}\left(\phi \underline{\tilde{u}}^{\bullet}\right)-\phi \tilde{\boldsymbol{\sigma}}^{\bullet}\right) \text { applied in } \omega_{2} \\
& \text { a body force } \tilde{\boldsymbol{\sigma}}^{\bullet} \underline{\operatorname{grad}}(\phi) \text { in } \omega_{2}
\end{aligned}
$$

The computation of the stress intensity factors and the estimation procedure for the error in the stress intensity factors can be summarized as

1. Solve the initial problem to obtain a finite element solution $\underline{u}_{h}, \boldsymbol{\sigma}_{h}$.

2. Use formula (47) to calculate $K_{\mathrm{I}, h}$ and $K_{\mathrm{II}, h}$.

3. Calculate the error estimator $\hat{e}_{h}$ for the initial problem and the elementary contributions $\hat{e}_{h E}$.

4. Solve two auxiliary problems on the initial mesh with the loading defined by Equation (48).

5. Calculate the error estimators $\hat{e}_{h}^{\text {aux,I }}, \hat{e}_{h}^{\text {aux,II }}$ and the elementary contributions $\hat{e}_{h E}^{\text {aux,II }}, \hat{e}_{h E}^{\text {aux, II }}$ for the two auxiliary problems.

6. Calculate the error estimators for $K_{\mathrm{I}}$ and $K_{\mathrm{II}}$

$$
\begin{gathered}
\hat{\varepsilon}_{\mathrm{I}, \mathrm{loc}}=\sum_{E} \hat{e}_{h E} \hat{e}_{h E}^{\mathrm{aux}, \mathrm{I}} \\
\hat{\varepsilon}_{\mathrm{II}, \mathrm{loc}}=\sum_{E} \hat{e}_{h E} \hat{e}_{h E}^{\mathrm{aux}, \mathrm{II}}
\end{gathered}
$$

7. Calculate the estimated bounds:

$$
\begin{gathered}
\hat{K}_{\mathrm{I}, \text { up }}=K_{\mathrm{I}, \mathrm{h}}+\hat{\varepsilon}_{\mathrm{I}, \mathrm{loc}} \\
\hat{K}_{\mathrm{I}, \mathrm{lo}}=K_{\mathrm{I}, \mathrm{h}}-\hat{\varepsilon}_{\mathrm{I}, \mathrm{loc}} \\
\hat{K}_{\mathrm{II}, \text { up }}=K_{\mathrm{II}, \mathrm{h}}+\hat{\varepsilon}_{\mathrm{II}, \mathrm{loc}} \\
\hat{K}_{\mathrm{II}, \mathrm{lo}}=K_{\mathrm{II}, \mathrm{h}}-\hat{\varepsilon}_{\mathrm{II}, \mathrm{loc}}
\end{gathered}
$$

\section{NUMERICAL EXAMPLES}

Three examples will be presented. The first problem is that of a single edge crack in a plate subjected to a uniform tension state (Figure 4). The second problem is that of a cantilever beam subjected to end shear, with the same geometry as the first problem, but involving a combination of Modes I and II (Figure 5). The third example is that of a rectangular plate with an inclined crack, as shown in Figure 6. The geometric parameters are $w=7, a / w=\frac{1}{2}$, $L / w=\frac{16}{7}$ for Problems 1 and 2 , and $w=7, a / w=\frac{1}{2}, L_{1} / w=L_{2} / w=1, \phi=60^{\circ}$ for Problem 3 . The Young's modulus is $E=210000 \mathrm{MPa}$, and Poisson's ratio is $v=0.25$. All the numerical 


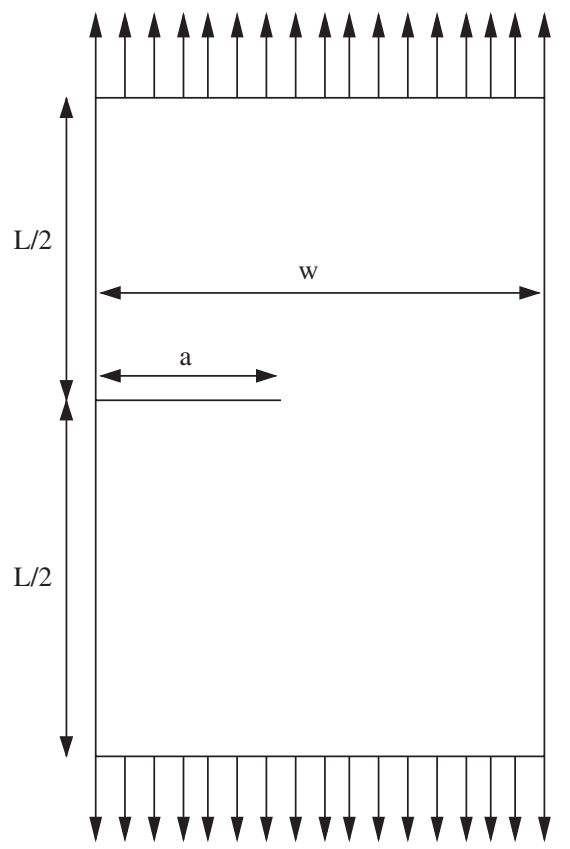

Figure 4. Problem 1.

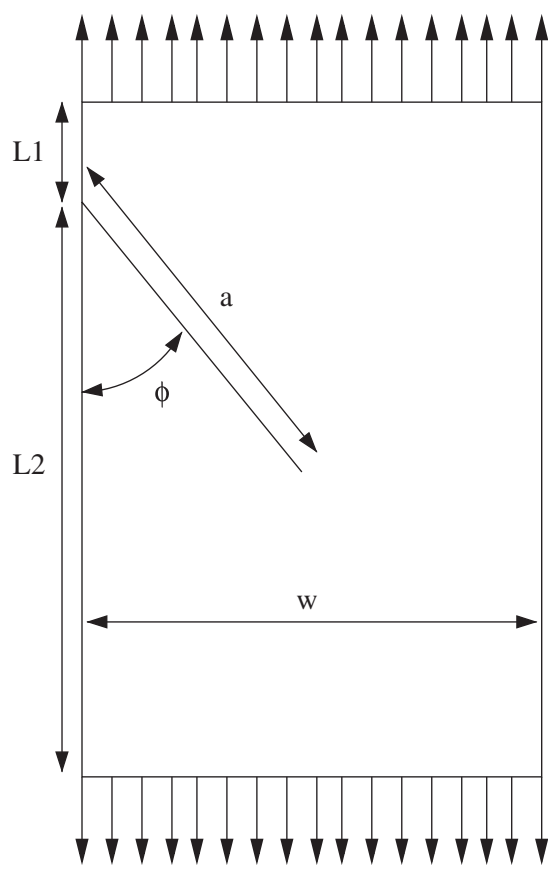

Figure 6. Problem 3.

Copyright (c) 2006 John Wiley \& Sons, Ltd.

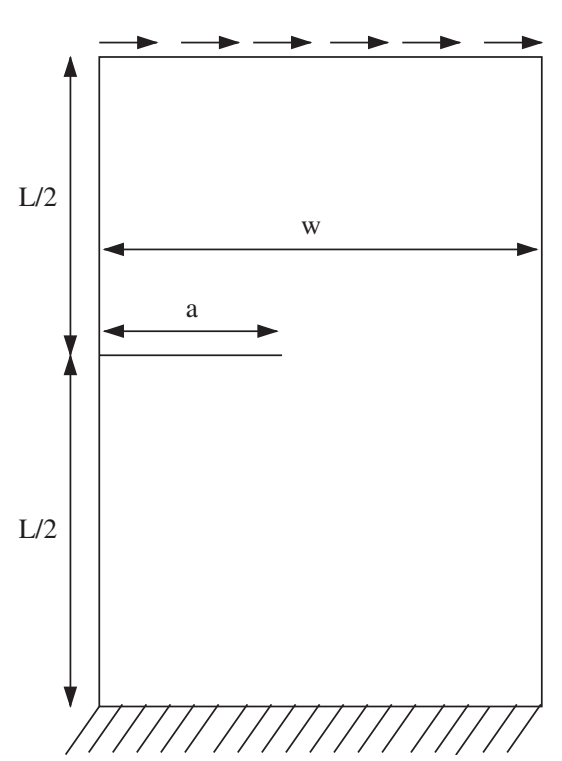

Figure 5. Problem 2.

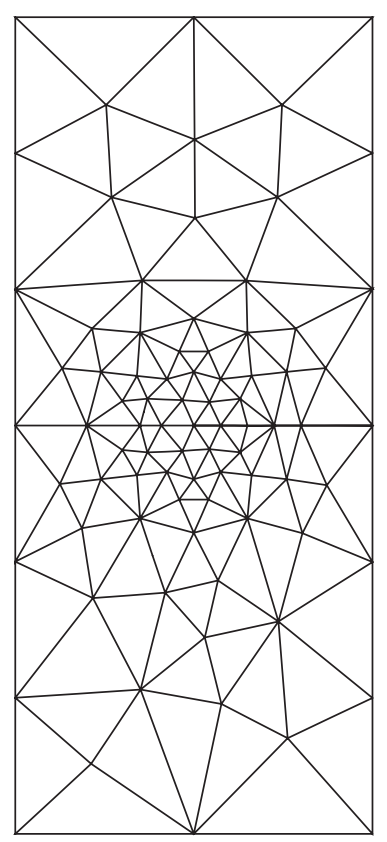

Figure 7. Problem 1: coarse mesh.

Int. J. Numer. Meth. Engng 2006; 68:299-316 


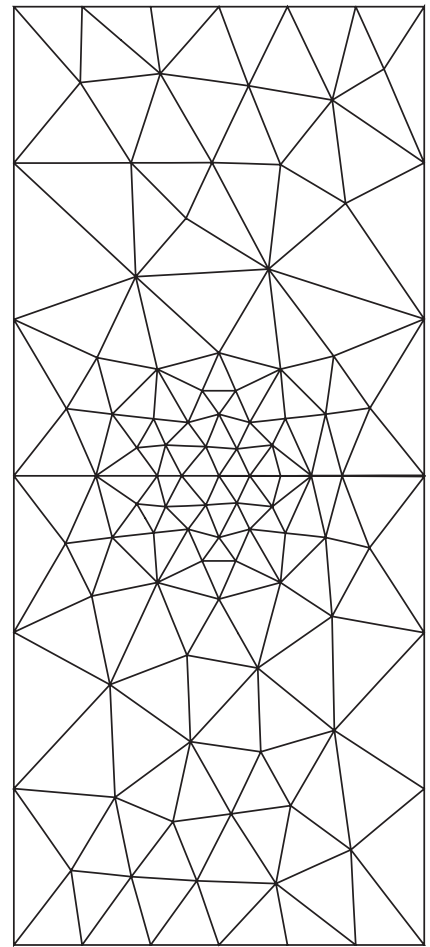

Figure 8. Problem 2: coarse mesh.

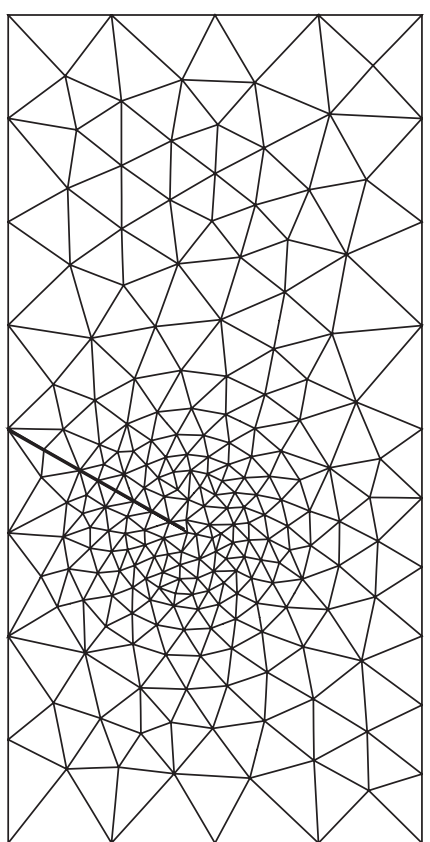

Figure 9. Problem 3: coarse mesh.

results were obtained using six-node triangular elements. The coarsest meshes are shown in Figures 7-9. In each case, we studied the evolution of the stress intensity factors as a function of the number of degrees of freedom. For each example, the reference stress intensity factors were obtained on a very refined mesh with more than 100000 elements, which we call the 'truth' mesh; these are denoted $K_{\mathrm{I}, \text { ref }}$ and $K_{\mathrm{II} \text {,ref }}$.

For the first problem, only the open-mode stress intensity factor $K_{\mathrm{I}}$ was studied since the shear-mode stress intensity factor equals zero. $K_{\text {I,ref }}$ calculated on the truth mesh was 9.335 . The relative difference between this value and that given in Reference [25], $K_{\mathrm{I}}=9.37$, is very small $(0.37 \%)$, which assesses the good quality of the extraction technique. The values obtained for the stress intensity factors as functions of the number of the degree of freedom of the mesh being used are given in Table I.

In the second problem, both open- and shear-mode stress intensity factors are present. The stress intensity factors $K_{\mathrm{I} \text {,ref }}$ and $K_{\mathrm{II} \text {,ref }}$ obtained on the truth mesh were 33.93 and 4.530 , respectively. The stress intensity factors given in Reference [19] for the open mode and in Reference [25] for the shear mode are 34.0 and 4.49. The relative differences are $0.21 \%$ for the open mode and $0.89 \%$ for the shear mode. The stress intensity factors and estimated errors calculated for each mesh are given in Table II for the open mode and in Table III for the shear mode. 
Table I. Problem 1: calculated stress intensity factors and their bounds.

\begin{tabular}{lrrrr}
\hline Number of DOFs & \multicolumn{1}{c}{674} & 1454 & 5706 & 22604 \\
\hline$K_{\mathrm{I}, h}$ & 8.813 & 9.040 & 9.177 & 9.244 \\
$\varepsilon_{\mathrm{I}, \mathrm{loc}}$ & 2.322 & 1.718 & 0.621 & 0.435 \\
$\hat{K}_{\mathrm{I} \text {,up }}$ & 11.136 & 10.758 & 9.798 & 9.679 \\
$\hat{K}_{\mathrm{I}, \text { lo }}$ & 6.491 & 7.322 & 8.556 & 8.809 \\
$K_{\mathrm{I}, \text { ref }}$ & 9.335 & 9.335 & 9.335 & 9.335 \\
\hline
\end{tabular}

Table II. Problem 2: open-mode stress intensity factors and their bounds.

\begin{tabular}{lcccc}
\hline Number of DOFs & 774 & 1742 & 6826 & 27026 \\
\hline$K_{\mathrm{I}, h}$ & 32.04 & 32.86 & 33.36 & 33.61 \\
$\varepsilon_{\mathrm{II}, \text { loc }}$ & 8.482 & 6.275 & 3.168 & 1.579 \\
$\hat{K}_{\mathrm{I}, \text { up }}$ & 40.53 & 39.13 & 36.53 & 35.19 \\
$\hat{K}_{\mathrm{I}, \mathrm{lo}}$ & 23.56 & 26.58 & 30.19 & 32.03 \\
$K_{\mathrm{I} \text {,ref }}$ & 33.93 & 33.93 & 33.93 & 33.93 \\
\hline
\end{tabular}

Table III. Problem 2: shear-mode stress intensity factors and their bounds.

\begin{tabular}{lcccl}
\hline Number of DOFs & 774 & 1742 & 6826 & 27026 \\
\hline$K_{\text {II }, h}$ & 4.363 & 4.475 & 4.490 & 4.499 \\
$\varepsilon_{\text {I,loc }}$ & 2.358 & 1.658 & 0.7860 & 0.3786 \\
$\hat{K}_{\text {II, up }}$ & 6.721 & 6.133 & 5.276 & 4.878 \\
$\hat{K}_{\text {II,lo }}$ & 2.004 & 2.818 & 3.704 & 4.120 \\
$K_{\text {II,ref }}$ & 4.530 & 4.530 & 4.530 & 4.530 \\
\hline
\end{tabular}

Table IV. Problem 3: open-mode stress intensity factors and their bounds.

\begin{tabular}{lcccc}
\hline Number of DOFs & 1790 & 4472 & 7834 & 22274 \\
\hline$K_{\mathrm{I}, h}$ & 6.160 & 6.263 & 6.287 & 6.314 \\
$\varepsilon_{\mathrm{I}, \text { loc }}$ & 0.667 & 0.366 & 0.417 & 0.245 \\
$\hat{K}_{\mathrm{I} \text {,up }}$ & 6.827 & 6.630 & 6.704 & 6.559 \\
$\hat{K}_{\mathrm{I}, \text { lo }}$ & 5.492 & 5.897 & 5.870 & 6.069 \\
$K_{\mathrm{I}, \text { ref }}$ & 6.338 & 6.338 & 6.338 & 6.338 \\
\hline
\end{tabular}

Table V. Problem 3: shear-mode stress intensity factors and their bounds.

\begin{tabular}{lcccc}
\hline Number of DOFs & 1790 & 4472 & 7834 & 22274 \\
\hline$K_{\text {II }, h}$ & 1.905 & 1.883 & 1.882 & 1.868 \\
$\varepsilon_{\text {II,loc }}$ & 0.153 & 0.091 & 0.089 & 0.058 \\
$\hat{K}_{\text {II, up }}$ & 2.058 & 1.974 & 1.971 & 1.926 \\
$\hat{K}_{\text {II,lo }}$ & 1.753 & 1.792 & 1.793 & 1.811 \\
$K_{\text {II,ref }}$ & 1.860 & 1.860 & 1.860 & 1.860 \\
\hline
\end{tabular}




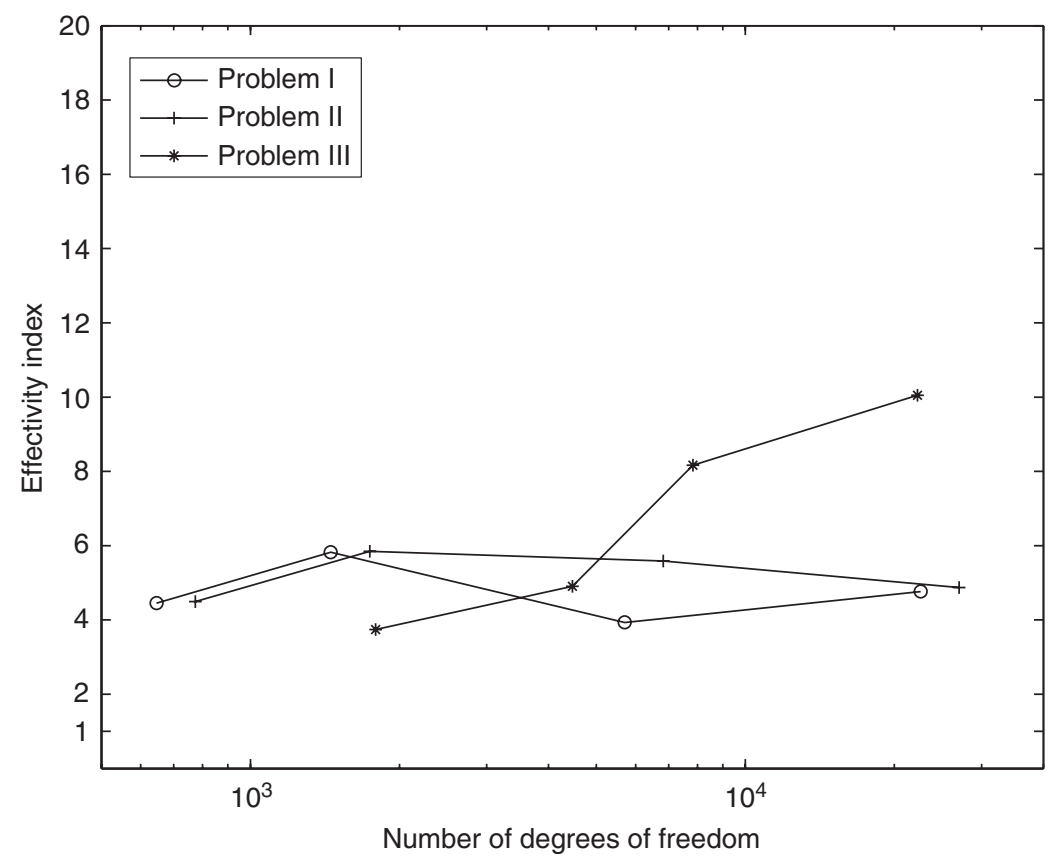

Figure 10. Effectivity index $K_{\mathrm{I}}$.

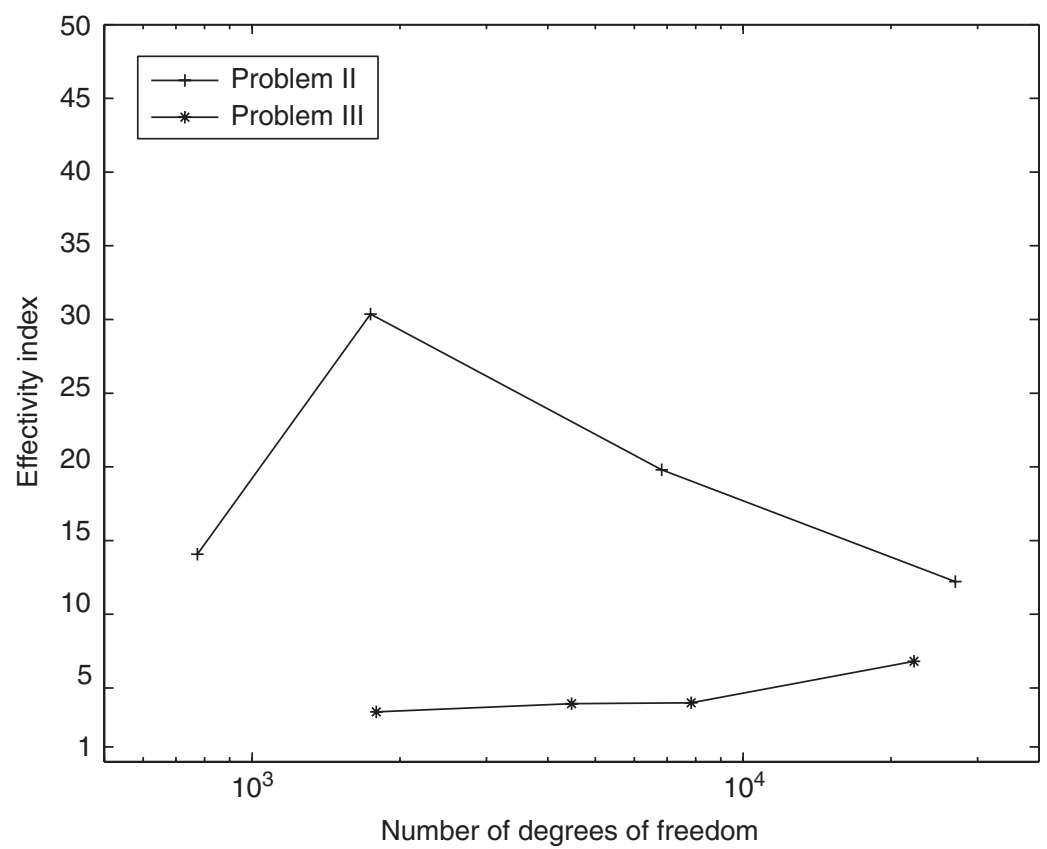

Figure 11 . Effectivity index $K_{\mathrm{II}}$. 
In the third problem, the reference stress intensity factors obtained with the truth mesh were $K_{\mathrm{I} \text {,ref }}=6.338$ and $K_{\mathrm{II}, \text { ref }}=1.860$. The relative difference between these and the values given in Reference [25], $K_{\mathrm{I}}=6.63$ and $K_{\mathrm{II}}=1.91$, is sufficiently small (4.4\% for the open mode and $2.6 \%$ for the shear mode). The stress intensity factors and the estimated error are given in Table IV for the open mode and in Table V for the shear mode.

We also calculated the effectivity indexes defined as $\eta_{\bullet}=\varepsilon_{\bullet}$, loc $/ \mid K_{\bullet}, \mathrm{h}-K_{\bullet}$,ref $\mid$ with $\bullet=\mathrm{I}$, II. The evolutions of these effectivity indexes are shown in Figure 10 for the open mode and in Figure 11 for the shear mode.

\section{CONCLUSION}

We presented an error estimator for stress intensity factors in elastic fracture mechanic. The calculation of the open- and shear-mode stress intensity factors requires the resolution of a single finite element problem with three different loading cases. The results of these calculations are used to calculate the error estimator. Numerical experiments showed that the proposed error estimator leads to usable lower and upper bounds of the quantities of interest.

\section{REFERENCES}

1. Babuška I, Rheinboldt WC. A posteriori estimates for the finite element method. International Journal for Numerical Methods in Engineering 1978; 12:1597-1615.

2. Ladevèze P, Pelle JP. Méthode de calcul par encadrement des fréquences propres de structures élastiques. Comptes Rendus de l'Académie des Sciences Série II. Paris 1983; 296:1757-1760.

3. Zienkiewicz OC, Zhu JZ. A simple error estimator and adaptive procedure for practical engineering analysis. International Journal for Numerical Methods in Engineering 1987; 24:337-357.

4. Babuška I, Miller A. The post-processing approach of the finite element method part ii: The calculation of the stress intensity factor. International Journal for Numerical Methods in Engineering 1982; 20:1111-1129.

5. Kelly DW, Isles JD. Procedures for residual equilibration and local error estimation in the finite element method. Communications in Applied Numerical Methods 1989; 5:497-505.

6. Rannacher R, Stuttmeier FT. A feedback approach to error control in finite element methods: application to linear elasticity. Computational Mechanics 1997; 19:434-446.

7. Rannacher R, Stuttmeier FT. A posteriori error control and mesh adaptation for f.e. models in elasticity and elastoplasticity. In Advances in Adaptive Computational Methods in Mechanics, Ladevèze P, Oden JT (eds), Studies in Applied Mechanics, vol. 47. Elsevier: Amsterdam, 1998; 275-292.

8. Peraire J, Patera A. Bounds for linear-functional outputs of coercive partial differential equations: local indicators and adaptive refinement. In Advances in Adaptive Computational Methods, Ladevèze P, Oden JT (eds). Elsevier: Amsterdam, 1998; 199-216.

9. Prudhomme S, Oden JT. On goal-oriented error estimation for elliptic problems: application to the control of pointwise errors. Computer Methods in Applied Mechanics and Engineering 1999; 176:313-331.

10. Ladevèze P, Rougeot P, Blanchard P, Moreau JP. Local error estimators for finite element analysis. Computer Methods in Applied Mechanics and Engineering 1999; 176:231-246.

11. Ohnimus S, Stein E, Walhorn E. Local error estimates of fem for displacements and stresses in linear elasticity by solving local neumann problems. International Journal for Numerical Methods in Engineering 2001; 52:727-746.

12. Prudhomme S, Oden JT, Westermann T, Bass J, Botkin ME. Practical methods for a posteriori error estimation in engineering applications. International Journal for Numerical Methods in Engineering 2003; 56:1193-1224.

13. Strouboulis T, Babuška I, Datta DK, Copps K, Gangaraj SK. A posteriori estimation and adaptative control of the error in the quantity of interest. part 1: A posteriori estimation of the error in the von mises stress and the stress intensity factor. Computer Methods in Applied Mechanics and Engineering 2000; 180:261-274. 
14. Heintz P, Larsson P, Hansbo P, Runesson K. On adaptive strategies and error control in fractures mechanics. Technical Report, Chalmers Finite Element Center, Chalmers University of Technology, 2002, preprint 2002-14.

15. Heintz P, Samuelsson K. On adaptive strategies and error control in fracture mechanics. Computers and Structures 2004; 82:485-497.

16. Ruter M, Stein E. Goal-oriented a posteriori error in linear elastic fracture mechanics. Computer Methods in Applied Mechanics and Engineering 2006; 195:251-278.

17. Xuan ZC, Parés N, Peraire J. Computing upper and lower bounds for the $J$-integral in two-dimensional linear elasticity. Computer Methods in Applied Mechanics and Engineering 2006; 195:430-443.

18. Sih GC, Rice JR. The bending of plates of dissimilar materials with crack. Journal of Applied Mechanics 1964; 477-482.

19. Stern M, Becker EB, Dunham RS. A contour integral computation of mixed-mode stress intensity factors. International Journal of Fracture 1976; 12(3):359-368.

20. Ladevèze P, Pelle JP, Rougeot P. Error estimation and mesh optimization for classical finite elements. Engineering Computation 1991; 8:69-80.

21. Ladevèze P, Rougeot P. New advances on a posteriori error on constitutive relation in f.e. analysis. Computer Methods in Applied Mechanics and Engineering 1997; 150:239-249.

22. Florentin E, Gallimard L, Pelle JP. Evaluation of the local quality of stresses in $3 \mathrm{~d}$ finite element analysis. Computer Methods in Applied Mechanics and Engineering 2002; 191:4441-4457.

23. Prager W, Synge JL. Approximation in elasticity based on the concept of functions space. Quarterly of Applied Mathematics 1947; 5:261-269.

24. Florentin E, Gallimard L, Pelle JP. Etude de la qualité locale de différentes versions de l'estimateur d'erreur en relation de comportement. Revue européenne des éléments finis 2003; 12(6):761-783.

25. Murakami Y. Stress Intensity Factors Handbook. Pergamon Press: Oxford, 1986. 